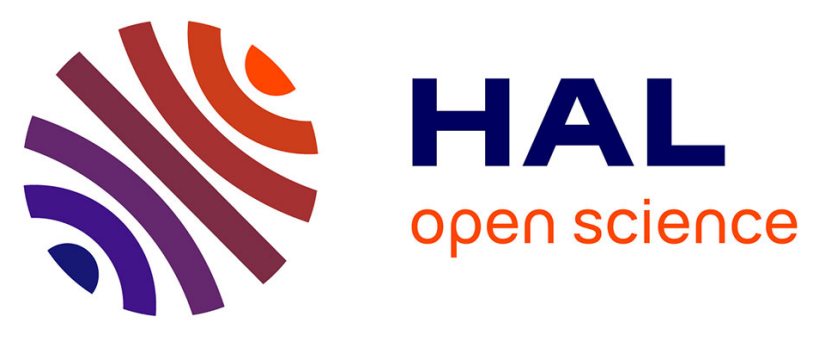

\title{
Relations entre la fertilité pollinique et les gènes de résistance au Fusarium oxysporum f. sp. lycopersici chez la tomate. Conséquences pour la sélection de variétés résistantes
}

Henri Laterrot, Brigitte Maisonneuve, Hélène Thomas

\section{To cite this version:}

Henri Laterrot, Brigitte Maisonneuve, Hélène Thomas. Relations entre la fertilité pollinique et les gènes de résistance au Fusarium oxysporum f. sp. lycopersici chez la tomate. Conséquences pour la sélection de variétés résistantes. Agronomie, 1984, 4 (10), pp.993-997. hal-00884603

\section{HAL Id: hal-00884603 https://hal.science/hal-00884603}

Submitted on 1 Jan 1984

HAL is a multi-disciplinary open access archive for the deposit and dissemination of scientific research documents, whether they are published or not. The documents may come from teaching and research institutions in France or abroad, or from public or private research centers.
L'archive ouverte pluridisciplinaire HAL, est destinée au dépôt et à la diffusion de documents scientifiques de niveau recherche, publiés ou non, émanant des établissements d'enseignement et de recherche français ou étrangers, des laboratoires publics ou privés. 


\title{
Relations entre la fertilité pollinique et les gènes de résistance au Fusarium oxysporum $f$. sp. lycopersici chez la tomate. Conséquences pour la sélection de variétés résistantes
}

\author{
Henri LATERROT \& Brigitte MAISONNEUVE (*) \\ avec la collaboration technique de Hélène THOMAs \\ I.N.R.A., Station d'Amélioration des Plantes maraîchères, Centre de Recherches d'Avignon, B.P. 94, \\ F 84140 Montfavet
}

RÉSUMÉ

\begin{abstract}
La qualité du pollen (estimée par le pourcentage de grains d'aspect normal après coloration au carmin acétique) a été étudiée chez des hybrides hétérozygotes pour le gène I, le gène I-2 ou les 2 gènes de résistance au Fusarium. Nos résultats s'interprètent de façon cohérente si l'on admet la liaison entre I et le facteur gamétophytique X fonctionnant selon le mécanisme décrit par AleXANDER (1973). Ainsi 9 hybrides hétérozygotes pour I ne produisent que 55 à 71 p. 100 de grains de pollen normaux. Le facteur $\mathrm{X}$ diminue aussi la quantité de pollen de 40 p. 100 quand il est à l'état hétérozygote. Il est également confirmé que I n'est pas lié à X chez « Campbell 1327 ». Des lignées possédant I-2 sans I ont été obtenues; chez celles-ci, I-2 n'est pas lié à X.

En conséquence, et en tenant compte de l'extension de la race I de Fusarium, les meilleures combinaisons pour les hybrides $\mathrm{F} 1$ commerciaux destinés aux cultures abritées sont, par ordre de préférence :

- soit l'hybridation entre une lignée possédant $\mathrm{I}$ associé à $\mathrm{X}$ et une lignée portant les 2 gènes I et $\mathrm{I}-2$ associés à $\mathrm{X}$;

- soit l'hybridation entre une lignée possédant I issu de "Campbell 1327 » et une lignée portant I-2 sans I. En conséquence annexe, une observation du pollen après coloration permet en cours de sélection de repérer facilement et avec peu d'erreurs les plantes hétérozygotes pour I associé à X.
\end{abstract}

Mots clés additionnels : Lycopersicon esculentum, qualité du pollen, quantité de pollen, facteur gamétophytique.

Relations between pollen fertility and Fusarium oxysporum f. sp. lycopersici in tomato. Consequences for breeding resistant varieties.

The pollen quality of F1 hybrids heterozygous for Fusarium resistance (I gene, I-2 gene or I and I-2 genes) was studied. The percentage of normal grains was measured after aceto-carmine staining. The effect of $X$ gametophytic factor on $\mathrm{X}^{+}$pollen grain abortion in $\mathrm{X} / \mathrm{X}^{+}$plants and the linkage between $\mathrm{X}$ and I genes were confirmed. Indeed, only 55 to $71 \%$ of pollen grains from 9 heterozygous hybrids ( $\left(\mathrm{I} / \mathrm{I}^{+}\right)$was normal, while 85 to $99 \%$ of pollen grains from homozygous genotype $\left(\mathrm{I} / \mathrm{I}\right.$ or $\left.\mathrm{I}^{+} / \mathrm{I}^{+}\right)$was normal. When the $\mathrm{X}$ factor was heterozygous, pollen quantity also decreased by $40 \%$. The non-linkage between $X$ and I of "Campbell 1327 " was confirmed. Some lines with I-2 and without I were obtained; the heterozygous hybrid $(\mathrm{I}-2 / \mathrm{I}-2+)$ from these lines had good quality pollen and therefore I-2 and X were not linked in these genotypes.

According to the results, and to the incidence of race 1 of Fusarium, the best hybrid cultivars for protected cultivation would be :

- hybrid between one line with I linked to $\mathrm{X}$ and one line with both genes (I and I-2) linked to X ; - hybrid between one line with I from "Campbell 1327" and one line with I-2 not linked to X.

Another consequence is that pollen staining can be used during a breeding program to test which plant is heterozygous for I (linked to X) without a Fusarium resistance test of the offspring.

Additional key words : Lycopersicon esculentum, pollen quality, pollen quantity, gametophytic factor. 


\section{INTRODUCTION}

Les températures froides sont connues pour provoquer une mauvaise nouaison chez la tomate (Lycopersicon esculentum Mill.) due en grande partie à une baisse de fertilité pollinique (MAISONNEUVE \& PHILOUZE, 1982). D'autre part, dès la découverte de la résistance au Fusarium chez Lycopersicon pimpinellifolium Mill. "Accession 160 », il a été suggéré que le gène dominant $\mathrm{I}$, qui la contrôle, était lié à un facteur $\mathrm{X}$ affectant la valeur des microgamètes (BOHN \& TUCKER, 1940). Ultérieurement, les déviations de disjonction pour le gène I ont été confirmées (KEDAR et al., 1967 ; HONMA \& VRIESENGA, 1972). Ces déviations peuvent être expliquées par l'avortement d'une partie des microgamètes porteurs de $\mathrm{X}^{+}$chez les plantes hétérozygotes pour $X$ (ALEXANDER, 1973). Alors que les plantes homozygotes $\mathrm{X} / \mathrm{X}$ et $\mathrm{X}^{+} / \mathrm{X}^{+}$sont fertiles, les hybrides hétérozygotes $\mathrm{X} / \mathrm{X}^{+}$ne produisent qu'une quantité réduite de pollen fertile, ce qui entraîne parfois des problèmes de nouaison, surtout lorsqu'ils sont cultivés en serres froides, ou peu chauffées.

Une solution à ces problèmes peut être apportée par l'utilisation de « Campbell 1327 » qui possède l'allèle I non lié à X (PECAUT, 1976). Mais ce géniteur n'apporte que la résistance à la race $0\left(^{*}\right)$, et le gène $\mathrm{I}$, incomplètement dominant, n'assure pas toujours une protection suffisante à l'état hétérozygote.

Il a paru intéressant d'étudier si le gène I-2 de résistance au Fusarium, localisé comme X et I sur le chromosome 11 (LATERROT, 1976), mais non allèle de I, était également lié à X. Cette étude était rendue possible par l'obtention récente de lignées portant I-2 sans I (LATERrot \& Philouze, 1984).

Ainsi, nous avons étudié la qualité du pollen chez des hybrides hétérozygotes pour la résistance au Fusarium. De plus, la quantité de pollen a été mesurée chez quelques génotypes afin de savoir si l'utilisation des lignées riches en pollen comme parents d'hybrides permettait de compenser - en partie ou en totalité l'action négative de $\mathrm{X}$ sur la production de pollen.

(*) Nous suivons dans cet article les règles proposées par GABE (1975) pour la nomenclature des races : race 0 pour la race commune contrôlée par le gène I et race 1 pour la race non contrôlée par I (nommé aussi I-1) mais contrôlée par le gène I-2. Il convient de remarquer que des résultats récents (LATERrot \& PhILouze, 1984) montrent que le gène I-2 ne contrôle pas la race 0 .

\section{MATÉRIEL ET MÉTHODES}

\section{A. Matériel végétal}

Les lignées suivantes résistantes au Fusarium ont été étudiées :

- « Marporum », version de la variété «Supermarmande » portant le gène I introduit par rétrocroisements à partir de la lignée "Anahu » (LATERROT, 1975) ;

- « Campbell 1327 », variété portant le gène I non lié à X (PECAUT, 1976);

- «Ideucenzi », 6 lignées sœurs issues d'un croisement complexe où le gène I-2 provenant de « Florida MH1 » a été séparé de I (LATERrot \& Philouze, 1984) ;
- "Mobox », version de la variété «Monalbo" issue de 6 rétrocroisements réalisés depuis «Walter 742-R1-3-3-BK ", porteuse de I et I-2.

A ce matériel ont été ajoutées des lignées sensibles au Fusarium ("Supermarmande ", " Monalbo », " Apédice ") et différentes combinaisons hybrides F1 (tabl. 1).

\section{B. Méthodes}

L'expérience a été effectuée au cours de 4 essais (tabl. 1). Les plantes ont été cultivées en conditions favorables à la production de pollen, sous serre en verre chauffée (température minimale de $13-14{ }^{\circ} \mathrm{C}$ ) de manière à déceler l'effet de $\mathrm{X}$ plus spécifiquement qu'on ne pourrait le faire en serre froide ou peu chauffée.

En avril-mai 1982, les fleurs ont été prélevées sur 15 génotypes représentés chacun par 6 plantes en pleine terre. En octobre 1982, mars 1983 et septembreoctobre 1983, les fleurs ont été prélevées respectivement sur 10,3 et 5 génotypes, représentés chacun par 2 ou 3 plantes en pots.

La qualité du pollen, estimée par le pourcentage de grains d'aspect normal après coloration au carmin acétique, a été étudiée à 4 dates par culture, sur un mélange de pollen de fleurs récoltées à l'anthèse (pollen de 15 à 30 fleurs avec comptage de 2 fois 150 grains en avril-mai 1982 ; pollen de 4 à 10 fleurs dans les autres cultures et comptage de 1 ou 2 fois 150 grains).

De plus, lors de la $1^{\text {re }}$ culture en 1982 , des mesures de quantité de pollen récolté après vibration avec un vibreur électrique (MAISONNEUVE \& PHILOUZE, 1982) ont été effectuées.

\section{RÉSULTATS}

Les résultats des observations de la qualité du pollen sont rassemblés dans le tableau 1. Après avoir vérifié l'absence d'effet significatif (seuil 1 p. 100) entre dates de mesures, nous avons calculé la moyenne des mesures aux 4 dates sur les données transformées des pourcentages de grains normaux $(2 \operatorname{arc} \sin \sqrt{\text { p. 100) }}$.

Les 6 lignées "Ideucenzi » ne différant pas significativement entre elles, nous présentons donc la moyenne les concernant; il en est de même avec les 6 hybrides F1 entre ces lignées et « Monalbo ».

Nous observons une très bonne qualité de pollen (au moins 85 p. 100 de grains normaux) chez tous les génotypes homozygotes aux 2 loci des gènes I et I-2, à l'exception de la F1 (« Marporum » $\times$ « Campbell 1327 »). Chez les génotypes hétérozygotes pour le gène I, lorsque I est apporté par " Marporum » ou "Anahu ", la qualité du pollen varie de 55 à 71 p. 100 de grains normaux (fig. 1). En revanche, quand le gène I vient de «Campbell 1327 », le pollen est d'aussi bonne qualité chez les génotypes hétérozygotes que chez les génotypes homozygotes (aucune valeur n'est inférieure à 93 p. 100 de grains normaux) (fig. 1). De même, nous observons une bonne qualité du pollen lorsque le gène I-2 des lignées «Ideucenzi » est à l'état hétérozygote $(98$ p. 100 de grains normaux). 
TABLEAU 1

Qualité du pollen (p. 100 de grains de pollen normaux après coloration par le carmin acétique) d'une série de lignées sensibles ou résistantes au Fusarium, par les gènes I ou I-2, et de différentes combinaisons hybrides F1. Conclusions quant à la présence du facteur gamétophytique X.

Pollen quality (\% normal pollen grains after aceto-carmine staining) in several lines susceptible or resistant to Fusarium (I or I-2 genes) and in different $F 1$ hybrids. Conclusions for $X$ gametophytic factor.

\begin{tabular}{|c|c|c|c|c|c|c|}
\hline \multirow[b]{2}{*}{$\begin{array}{c}\text { Génotypes pour } \\
\text { I et I-2 }\end{array}$} & \multirow[b]{2}{*}{ Lignées ou hybrides F1 } & \multicolumn{4}{|c|}{$\%$ de grains normaux à différentes époques de mesure * } & \multirow[b]{2}{*}{$\begin{array}{l}\text { Conclusions } \\
\text { quant au } \\
\text { gène } X\end{array}$} \\
\hline & & $\begin{array}{l}\text { avril-mai } \\
1982\end{array}$ & $\begin{array}{l}\text { octobre } \\
1982\end{array}$ & $\begin{array}{l}\text { mars } \\
1983\end{array}$ & $\begin{array}{l}\text { sept.-oct. } \\
1983\end{array}$ & \\
\hline $\mathrm{I}^{+} / \mathrm{I}^{+}, \mathrm{I}-2^{+} / \mathrm{I}-2^{+}$ & 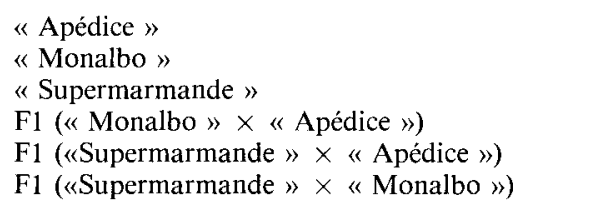 & $\begin{array}{l}98 \mathrm{ab} \\
98 \mathrm{abc} \\
97 \mathrm{abc} \\
98 \mathrm{abc} \\
99 \mathrm{a} \\
98 \mathrm{abc}\end{array}$ & $\begin{array}{l}- \\
98 \mathrm{a} \\
85 \mathrm{c} \\
- \\
-\end{array}$ & $\begin{array}{l}- \\
- \\
- \\
-\end{array}$ & $\begin{array}{l}- \\
98 \mathrm{a} \\
- \\
- \\
-\end{array}$ & $\begin{array}{c}+/+ \\
" \\
" \\
" \\
" \\
"\end{array}$ \\
\hline $\mathrm{I} / \mathrm{I}, \mathrm{I}-2^{+} / \mathrm{I}-2^{+}$ & $\begin{array}{l}\text { " Marporum } » \\
\text { «Campbell } 1327 » \\
\text { F1 (« Marporum » } \times \text { « Campbell } 1327 »)\end{array}$ & $\begin{array}{c}94 \mathrm{~cd} \\
- \\
-\end{array}$ & $95 \mathrm{ab}$ & $\begin{array}{l}97 \mathrm{a} \\
97 \mathrm{a} \\
72 \mathrm{~b}\end{array}$ & $\begin{array}{l}- \\
-\end{array}$ & $\begin{array}{l}X / X \\
+/+ \\
X /+\end{array}$ \\
\hline $\mathrm{I}^{+} / \mathrm{I}^{+}, \mathrm{I}-2 / \mathrm{I}-2$ & « Ideucenzi » (6 lignées) & - & - & - & 97 a & $+1+$ \\
\hline $\mathrm{I} / \mathrm{I}, \mathrm{I}-2 / \mathrm{I}-2$ & $\begin{array}{l}\text { «Flora Dade " } \\
\text { «Mobox» }\end{array}$ & $91 \bar{d}$ & $91 \mathrm{bc}$ & - & - & $\begin{array}{l}\mathrm{X} / \mathrm{X} \\
\mathrm{X} / \mathrm{X}\end{array}$ \\
\hline $\mathrm{I} / \mathrm{I}^{+}, \mathrm{I}-2^{+} / \mathrm{I}-2^{+}$ & 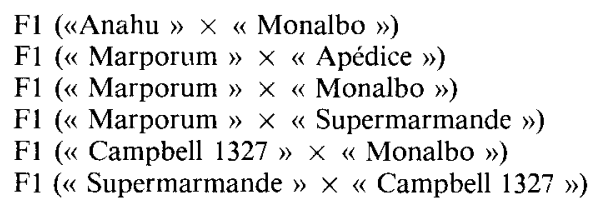 & $\begin{array}{l}71 \mathrm{e} \\
60 \mathrm{f} \\
65 \mathrm{ef} \\
- \\
-\end{array}$ & $\begin{array}{l}- \\
- \\
55 \mathrm{~d} \\
98 \mathrm{a} \\
93 \mathrm{ab}\end{array}$ & $\begin{array}{l}- \\
- \\
- \\
- \\
-\end{array}$ & $\begin{array}{l}61 \mathrm{~b} \\
- \\
- \\
9 \mathrm{a} \\
-\end{array}$ & $\begin{array}{l}\mathrm{X} /+ \\
" \\
" \\
" \\
+1+ \\
+1+\end{array}$ \\
\hline $\mathrm{I} / \mathrm{I}^{+}, \mathrm{I}-2 / \mathrm{I}-2^{+}$ & 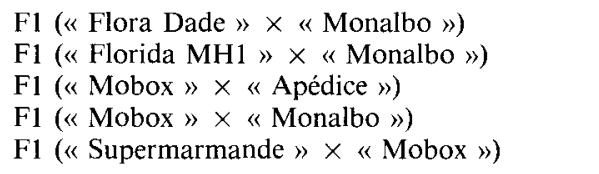 & $\begin{array}{l}- \\
70 \text { ef } \\
63 \text { ef } \\
68 \text { ef }\end{array}$ & $\begin{array}{c}61 \mathrm{~d} \\
- \\
59 \mathrm{~d} \\
-\end{array}$ & $\begin{array}{l}- \\
- \\
- \\
-\end{array}$ & $\begin{array}{l}\overline{50 \mathrm{c}} \\
\overline{-} \\
-\end{array}$ & $\begin{array}{l}\mathrm{X} /+ \\
" \\
» \\
" \\
"\end{array}$ \\
\hline $\mathrm{I}^{+} / \mathrm{I}^{+}, \mathrm{I}-2 / \mathrm{I}-2^{+}$ & F1 («Ideucenzi » $\times \ll$ Monalbo $»)$ & - & - & - & $98 \mathrm{a}$ & $+1+$ \\
\hline $\mathrm{I} / \mathrm{I}, \mathrm{I}-2 / \mathrm{I}-2^{+}$ & $\begin{array}{l}\text { F1 (« Marporum } » \times \ll \text { Mobox } ») \\
\text { F1 }(« \text { Mobox } » \times \ll \text { Campbell } 1327 »)\end{array}$ & $\begin{array}{l}95 \text { bcd } \\
-\end{array}$ & $\overline{57 \mathrm{~d}}$ & - & - & $\begin{array}{l}X / X \\
X /+\end{array}$ \\
\hline
\end{tabular}

* Les résultats suivis d'une même lettre pour une même époque de mesures ne diffèrent pas significativement au seuil de 1 p. 100 .

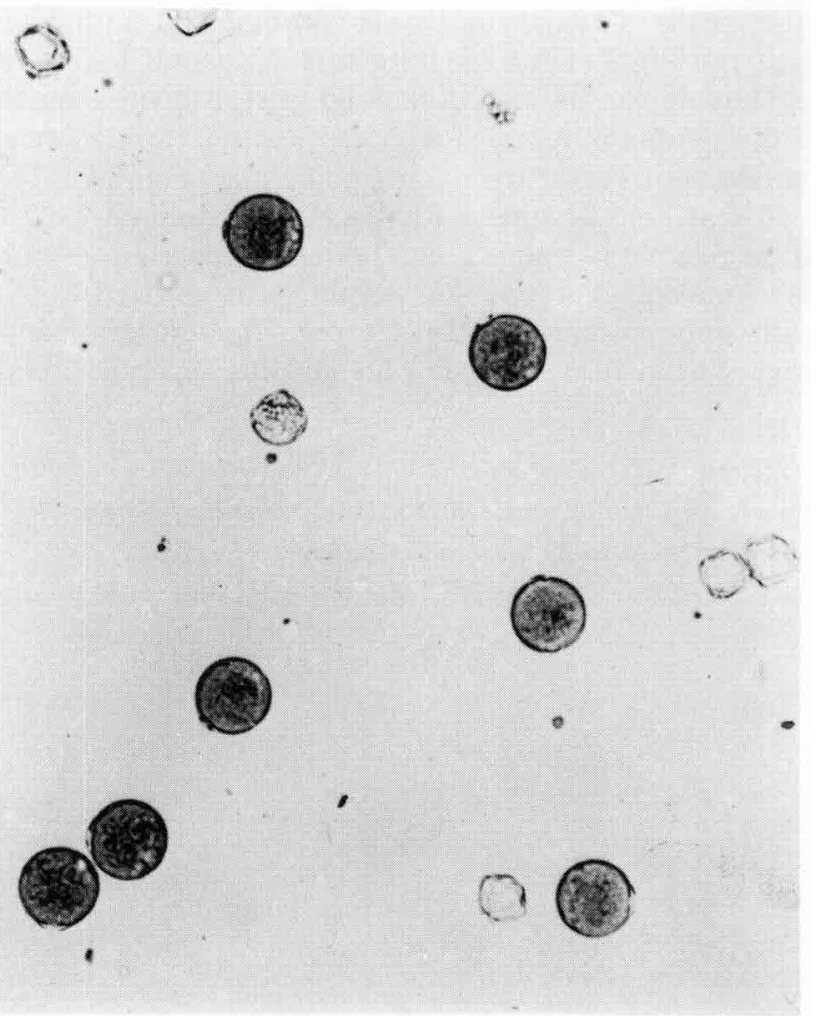

Le tableau 2 présente quelques valeurs de quantité de pollen produit par fleur. Les 2 hybrides hétérozygotes pour le gène I produisent moins de pollen que les génotypes, lignées ou hybrides, homozygotes pour ce gène.

\section{DISCUSSION}

Les résultats (tabl. 1) confirment donc que la liaison entre le gène $I$ de résistance à la race 0 de Fusarium et le facteur gamétophytique X (BOHN \& TUCKER, 1940) a été brisée dans la lignée «Campbell 1327 » (PECAUT, 1976). En effet, les hybrides (« Campbell 1327 » $\times$ " Monalbo ») et (“Supermarmande » $\times$ «Campbell $1327 »)$ ont un pollen d'aussi bonne qualité que celui des lignées parentales tandis que

Figure 1

Grains de pollen d'une plante $1 X / I^{+} X^{+}$après coloration par le carmin acétique :

- 7 grains d'aspect normal, ronds, lisses et colorés.

- 6 grains avortés, déformés, vides et non colorés.

Pollen grains from a $I X / I^{+} X^{+}$plant after aceto-carmin coloration :

-7 normal grains : round, unruffled and coloured.

-6 avorted grains : distorted, empty and uncoloured. 
l'hybride (« Marporum » $\times$ « Campbell 1327 ») a un pollen moins bon que celui de ses parents. Ces résultats sont en accord avec ceux que nous avons obtenus en étudiant des hybrides $\mathrm{F} 1$ commerciaux résistants au Fusarium (résultats non publiés) : ceux ayant un pollen de qualité moyenne (70 à 75 p. 100 de grains colorés au carmin acétique) sont hétérozygotes pour I et les autres (95 à 100 p. 100 de grains normaux) sont homozygotes pour I non issu de "Campbell 1327 » ou possèdent $I$ issu de « Campbell 1327 ». Une conséquence indirecte de ces résultats est qu'une estimation de la qualité du pollen produit dans un environnement favorable peut permettre de trier en partie les plantes hétérozygotes pour I non issu de «Campbell 1327 » de manière beaucoup plus rapide et simple qu'un test pour la résistance au Fusarium même si le risque d'erreurs, dues à des recombinaisons, n'est pas nul. De plus, dans une recherche de fixation de I, pour contrôler l'homozygotie de ce gène, il est nécessaire de tester pour la résistance au Fusarium un nombre plus élevé de plantes que ce qu'il est coutume d'observer dans le cas d'un caractère monogénique dominant (16 plantes avec $\mathrm{p} \leqslant 0,01)$.

Les résultats des lignées «Ideucenzi » (issues d'un hybride avec la lignée " Florida MH1 " (LATERROT \& PhilouzE, 1984) qui possède I, I-2 et X) et de leurs hybrides avec «Monalbo » montrent que I-2 n'est pas lié à $\mathrm{X}$ dans ce matériel qui est actuellement le seul disponible portant I-2 sans I. Ceci est en accord avec la localisation de I-2 sur le chromosome 11 , selon la séquence X-I - I-2 proposée par LATERROT (1976). Il semble donc que la liaison entre ces 3 gènes ait été brisée entre I et I-2. L'obtention de ces lignées ne permet cependant pas d'envisager l'utilisation de I-2 à l'état hétérozygote dans des hybrides commerciaux en raison de l'absence de la résistance à la race 0 , sinon en croisement avec un géniteur dont le gène I serait issu de «Campbell 1327 ». Il paraît évident que les lignées I-2, connues précédemment («Florida MH1 », «Flora Dade », « Mobox », entre autres) possèdent les 3 gènes $X$, I et $I-2$.

Si l'on admet que I est lié à X (sauf chez «Campbell 1327 ») et que I-2 n'est pas lié à X (chez «Ideucenzi »), les résultats montrent chez les hétérozygotes $\mathrm{X} / \mathrm{X}^{+}$un effet de $\mathrm{X}$ sur la microsporogenèse avec un minimum de 30 p. 100 de grains mal formés, ce qui correspond à l'hypothèse de l'effet de X sur l'avortement d'une grande partie des microgamètes porteurs de $\mathrm{X}^{+}$(ALEXANDER, 1973 ; RABINOVITCH et al.,
1978). L'absence d'anomalies au cours de la méiose (VRIESENGA \& HONMA, 1971) et l'aspect des grains observés (fig. 1) conduisent à l'hypothèse d'un avortement au cours de la mitose pollinique.

Les quelques mesures de quantité de pollen (tabl. 2) doivent être examinées avec précaution car la morphologie des fleurs est très différente selon les génotypes utilisés et ces mesures sont toujours délicates. L'effet de $\mathrm{X}$ se manifeste intensément chez les 2 hybrides hétérozygotes pour $\mathrm{I}$, avec une production de pollen qui n'atteint que 60 à 65 p. 100 de celle des lignées parentales; de même, la production de pollen de l'hybride ( Marporum» $\times$ « Apédice ») n'atteint que 60 p. 100 de celle de l'hybride («Supermarmande » $x$ «Apédice »). La quantité de pollen produite par ces hybrides est cependant assez voisine de celle obtenue à température optimale chez des lignées moyennes productrices de pollen (MAISONNEUVE \& PHILOUZE, 1982). Ainsi, la présence du facteur gamétophytique $\mathrm{X}$ à l'état hétérozygote ramène les hybrides très bons producteurs de pollen au niveau de lignées moyennes productrices. Il peut donc s'ensuivre une insuffisance de la quantité de pollen produit en culture froide ou peu chauffée.

\section{CONCLUSIONS}

Ces résultats apportent quelques idées importantes pour la sélection d'hybrides commerciaux. Le gène I-2 dans le seul matériel où il a été séparé de I n'est pas lié à $\mathrm{X}$. L'emploi du gène $\mathrm{I}$ à l'état homozygote qui, sur un autre plan, assure un niveau plus élevé de résistance qu'à l'état hétérozygote, semble indispensable pour avoir du pollen de bonne qualité et pour augmenter ainsi les chances d'avoir une bonne nouaison en conditions climatiques rigoureuses. Ceci n'est pas nécessaire si on utilise un géniteur portant $I$ sans $X$ issu de «Campbell 1327 ». Le sélectionneur devra aussi veiller à ne pas utiliser la combinaison d'un géniteur portant $I$ et $X$ avec un géniteur portant $I$ sans $X$.

Dans le cas de sélection pour la résistance à partir d'un géniteur portant $I$ avec $X$, un test rapide sur le pollen peut permettre de reconnaitre les plantes $\mathrm{I} / \mathrm{I}^{+}$.

L'état hétérozygote de I issu de « Campbell 1327 » n'est pas défavorable à une bonne production de pollen ; mais le génotype ${\mathrm{I} / \mathrm{I}^{+}}^{+}$semble parfois être insuffisant pour garantir un haut niveau de résistance à la race 0 et la race 1 est de plus en plus répandue dans

TABLEAU 2

Quantité de pollen (mg/fleur) de lignées sensibles ou résistantes au Fusarium (gène I lié à $X$ ) et de différentes combinaisons hybrides Fl. Pollen quantity (mg/flower) in Fusarium susceptible or resistant ( $I$ gene linked to $X$ ) lines and in different F1 hybrids.

\begin{tabular}{|c|c|c|c|}
\hline $\begin{array}{l}\text { Génotypes } \\
\text { pour IX }\end{array}$ & Lignées ou hybrides $F 1$ & \multicolumn{2}{|c|}{$\begin{array}{l}\text { Quantité de pollen * } \\
\text { (mg/fleur) }\end{array}$} \\
\hline $\mathbf{I}^{+} \mathbf{X}^{+} / \mathbf{I}^{+} \mathrm{X}^{+}$ & $\begin{array}{l}\text { «Supermarmande » } \\
\text { "Apédice } " \\
\text { F1 (« Supermarmande » } \times \text { « Apédice })\end{array}$ & $\begin{array}{l}2,35 \\
1,87 \\
1,93\end{array}$ & $\begin{array}{l}\mathrm{a} \\
\mathrm{b} \\
\mathrm{b}\end{array}$ \\
\hline $\mathrm{IX} / \mathrm{IX}$ & «Marporum » & 1,91 & $b$ \\
\hline $\mathrm{IX} / \mathbf{I}^{+} \mathrm{X}^{+}$ & $\begin{array}{l}\text { F1 (« Marporum » } \times \text { « Supermarmande ») } \\
\text { F1 (« Marporum » } \times \ll \text { Apédice ») }\end{array}$ & $\begin{array}{l}1,50 \\
1,16\end{array}$ & $\begin{array}{l}c \\
d\end{array}$ \\
\hline
\end{tabular}

* Les résultats suivis d'une même lettre ne diffèrent pas significativement au seuil de 1 p. 100. 
les cultures abritées. L'état hérérozygote de I-2 issu de "Ideucenzi " n'est pas non plus défavorable à une bonne production de pollen; mais le gène I-2 seul ne contrôle pas la race 0 de Fusarium. D'autre part, les 2 gènes réunis à l'état homozygote paraissent défavorables à la quantité de pollen (résultat de " Mobox », lignée X - I - I-2, non présentés ici). En conséquence, pour la création d'hybrides $\mathrm{F} 1$ destinés à la culture sous abris peu ou non chauffés, il semble prudent de choisir l'un des 2 génotypes suivants :

- de préférence : homozygote pour I et X et hétérozygote pour I-2 ;

- ou à la rigueur : hétérozygote pour $\mathrm{I}$ et $\mathrm{I}-2$ et homozygote pour $\mathrm{X}^{+}$.

Reçu le 18 janvier 1984 Accepté le 25 juin 1984

\section{RÉFÉRENCES BIBLIOGRAPHIQUES}

Alexander M. P., 1973. Investigation of the mode of action of the $\mathrm{X}$ locus in tomato. Euphytica, 22, 344-350.

Bohn G. W., Tucker C. M., 1940. Studies on Fusarium wilt of the tomato. I. Immunity in Lycopersicon pimpinellifolium Mill. and its inheritance in hybrids. Mo. Agric. Exp. Stn. Res. Bull,, 311, 1-82.

Gabe H. L., 1975. Standardization of nomenclature for pathogenic races of Fusarium oxysporum f. sp. lycopersici. Trans. Br. Mycol. Soc., 64 (1), 156-159.

Honma S., Vriesenga J. D., 1972. Inheritance of Fusarium resistance in a jointless tomato as affected by the $\mathrm{X}$ gametophytic factor. Euphytica, 21, 143-151.

Kedar N., Retig N., Katan J., 1967. Non-random segregation of gene I for Fusarium resistance in the tomato. Euphytica, 16, 258266.

Laterrot H., 1975. Séries de lignées isogéniques de tomate ne différant que par certains gènes de résistance aux maladies. Phytopathol. Mediterr., XIV, 2-3, 129-130.

Laterrot H., 1976. Localisation chromosomique de I-2 chez la tomate contrôlant la résistance au pathotype 2 de Fusarium oxysporum f. lycopersici. Ann. Amélior. Plantes, 26 (3), 485-491.

Laterrot H., Philouze J., 1984. Recombination between resistance to pathotype 1 (I-2 allele) and susceptibility to pathotype 0 ( $\mathrm{I}^{+}$ allele) of Fusarium oxysporum $f$. sp. lycopersici in tomato (Lycopersicon esculentum Mill.). Proc. Meet. of the Eucarpia Tomato Working Group, Wageningen (Pays-Bas), May 1984, 70-74.

Maisonneuve B., Philouze J., 1982. Action des basses températures nocturnes sur une collection variétale de tomate (Lycopersicon esculentum Mill.). II. Etude de la quantité et de la qualité du pollen. Agronomie, 2 (5), 453-458.

Pecaut P., 1976. Search of $I / I$ varieties without the gametophytic factor X. Rep. of Tomato Genet. Coop., 26, 10.

Rabinovitch H. D., Retig N., Kedar N., 1978. The mechanism of preferential fertilization in tomatoes carrying the I allele for Fusarium resistance. Euphytica, 27, 219-224.

Vriesenga J. D., Honma S., 1971. Investigation of the action of the X locus in the tomato. Euphytica, 20, 396-399. 